\title{
Localization of polysaccharides in isolated and intact cuticles of eucalypt, poplar and pear leaves by enzyme-gold labelling
}

Paula Guzmán ${ }^{1}$, Victoria Fernández ${ }^{1, *}$, María Luisa García ${ }^{2}$, Mohamed Khayet ${ }^{3}$, Agustín Fernández ${ }^{2}$, Luis Gil ${ }^{1}$

${ }^{1}$ Forest Genetics and Eco-physiology Research Group, School of Forest Engineering, Technical University of Madrid, Ciudad Universitaria s/n, 28040 Madrid, Spain

${ }^{2}$ Electron Microscopy National Centre, Faculty of Chemistry, University Complutense of Madrid, Avda. Complutense s/n, 28040 Madrid, Spain

${ }^{3}$ Department of Applied Physics I, Faculty of Physics, University Complutense of Madrid, Avda. Complutense s/n, 28040 Madrid, Spain

*Corresponding Author. E-mail: v.fernandez@upm.es, Phone: +34 913367113

\begin{abstract}
The presence and characteristics of cuticle polysaccharides have been demonstrated by staining and spectroscopic methods, but their location in the cuticle remains unclear. Furthermore, according to the prevailing model, polysaccharides are believed to be restricted to the cuticular layer and absent in the cuticle proper. With the aim of gaining insight into cuticular ultra-structure focussing on polysaccharides, cellulose and pectins have been identified and located in the transversal sections of isolated and intact adaxial leaf cuticles of Eucalyptus globulus, Populus x canescens and Pyrus communis by means of enzyme goldlabelling (Au-cellulase, EC 3.2.1.4, and -pectinase, EC 3.2.1.15) and transmission electron microscopy (TEM). The structure of the interface between the cuticle and the cell wall underneath was observed to influence the process of enzymatic isolation of leaf cuticles. Cellulose and pectins were detected for the first time in enzymatically isolated cuticles, sometimes appearing closely underneath the epicuticular wax layer. The location and presence of polysaccharides in intact and isolated leaf cuticles may have multiple implications, such as when estimating the bi-directional transport of substances between plant organs and the surrounding environment, or when interpreting organ ontogeny. The results are discussed within a plant ontological and ecophysiological context.
\end{abstract}

Keywords: Cellulose; Cuticle; Gold-labelling; Leaf; Pectins; Polysaccharides; Transmission electron microscopy 


\section{Introduction}

The outer surface of most aerial organs of plants such as leaves, flowers, fruits and nonwoody stems are covered with a composite, extracellular membrane, the cuticle, which plays a major physiological and structural role in the protection against multiple potential biotic and abiotic stress factors [1,2]. Due to the intimate relationship between the cuticle and the cell wall underneath, the cuticle may be considered a cutinized cell wall [3].

The concept of heterogeneity as a crucial component of cell wall structure and function [4,5] can be extended to the cuticle. From a chemical point of view, the cuticle is mainly composed of a cutin and/or cutan polymer matrix, waxes both embedded in (intracuticular) and deposited onto (epicuticular) the outer surface of such matrix, polysaccharides and phenolics [3,6].

According to the prevailing model [7], the cuticle consists of two different layers, the cuticle proper (CP) as the outermost region and the subjacent cuticular layer (CL). An epicuticular wax (EW) layer covers the $\mathrm{CP}$ and is in direct contact with the environment [8]. The CP, which is by definition free of polysaccharides, is the first cuticular region to appear during cuticle ontogeny, assembled to the primary cell wall by a pectic layer. The CL contains polysaccharides steaming from the epidermal cell wall and appears later during cell wall development [8].

Cellulose, hemicelluloses and pectins have been grossly isolated from tomato fruit cuticles, and they have been observed to play an important role on cuticle rheological properties [9]. The presence and characteristics of polysaccharides in leaf and fruit isolated cuticles examined as intact tissues or after cutin depolymerisation, have been analysed by different spectroscopic methods [e.g., 9-11]. Although it was found that cellulose fibres are mainly randomly oriented in cuticles of mature and ripe tomato fruits and Agave Americana young leaves $[9,10]$, the position and distinction between groups of polysaccharides cannot be ascertained by such analytical techniques. On the other hand, stains like osmium tetroxideuranyl acetate-lead citrate combination [12], calcofluor white [9], ruthenium red [13], periodic acid-thiosemicarbazide-silver proteinate tests [12,14] or chlor-zinc-iodine [15] have been used for detecting polysaccharides in cross-sections of isolated leaf and fruit cuticles by electron, optical and fluorescence microscopy. Nevertheless, the lack of specificity of the dyes, the potential degree of impregnation of polysaccharides with chiefly lipidic cuticular components, and the restrictions to observe stained tissues at higher magnifications by optical and fluorescence microscopy, pose an obstacle for elucidating the nature and location of polysaccharides in the cuticle.

Most of such constraints can be overcome by using gold $(\mathrm{Au})$-labelling techniques and transmission electron microscopy (TEM). Gold-enzyme labelling has proved to be a reliable tool for the identification and localization of selected polysaccharides in plant cell walls [e.g., 16,17]. Working with spruce leaf transversal sections and Au- enzymatic labelling, Tenberge [18] identified cellulose and hemicelluloses cuticle by, but failed to detect pectins.

In light of the current lack of information about the location of polysaccharides in leaf cuticles, we carried out enzymatic Au-labelling experiments to identify and locate cellulose and pectins in the adaxial leaf cuticle of three model plant species. European pear was selected since its leaf cuticle has been previously described [13]. The leaf cuticle of grey poplar has been analysed since it is a model species used for cuticular permeability studies [19]. The blue-gum eucalypt leaf has been examined due to its evergreen nature, markedly different eco-physiological habitat and commercial significance in forestry. 


\section{Materials and methods}

\subsection{Plant material}

Fully expanded leaves of Eucalyptus globulus Labill., Populus x canescens (Ait.) Sm. (P. alba L. x P. tremula L.) and Pyrus communis L. var. Blanca de Aranjuez, hereinafter referred to as eucalypt, poplar and pear, were selected for experimental purposes. Juvenile eucalypt leaves were collected from 1.5-year-old seedlings growing in the Forest Engineering School Arboretum (Technical University of Madrid, Spain). Poplar leaves were obtained from trees previously identified with molecular analyses grown in Losana (Soria, Spain; R Sierra, Personal communication). Pear leaves were collected from trees grown in the Royal Botanic Gardens of Madrid (CSIC, Spain). All the leaves were collected from medium size shoots during the summer.

Prior to isolating the cuticles enzymatically, leaf midveins and margins were removed with a scalpel. The enzymatic solution contained 2\% cellulase (EC 3.2.1.4, extracted from Trichoderma reesei, 10-20\% w/v; Celluclast ${ }^{\circledR}$, Novozymes, Bagsvared, Denmark), 2\% pectinase (EC 3.2.1.15, from Aspergillus aculeatus, 10\% w/v; Pectinex Ultra SP-L, Novozymes) plus $1 \%$ polyvinylpyrrolidone (Sigma-Aldrich, Munich, Germany) and $2 \mathrm{mM}$ sodium azide [20; with some modifications]. The $\mathrm{pH}$ was adjusted to 5.0 by adding sodium citrate. Eucalypt and pear leaves were maintained in the solution for one month, until the cuticle appeared to be fully separated from the underlying tissue, while poplar leaves remained in solution from one and a half to two months. Leaf tissues were digested at room temperature (23 to $25^{\circ} \mathrm{C}$ ), solutions were changed every two weeks and they were manually shaken at frequent time intervals. After the extraction period, clean intact adaxial cuticles were selected, thoroughly washed in deionized water, air-dried and stored for microscopic examination.

\subsection{Tissue fixation and embedding}

Enzymatically digested adaxial cuticles and fresh leaves were cut into about $4 \mathrm{~mm}^{2}$ pieces and subsequently fixed and embedded in LR-White resin, which is commonly used for immuno-cytochemistry and in relation to colloidal Au-labelling [21]. Samples were fixed in 1\% glutaraldehyde-4\% paraformaldehyde (both from Electron Microscopy Sciences (EMS), Hatfield, USA) for $4 \mathrm{~h}$ at $4^{\circ} \mathrm{C}$, rinsed in ice-cold phosphate buffer, $\mathrm{pH}$ 7.2, four times within a period of $6 \mathrm{~h}$ and left overnight. They were then dehydrated in an ethanol series of 30,50, 70, 80, 90, 95 and 100\% (x 2, 15 min each concentration) and embedded in ethanol-LR-White resin (London Resin Company, London, UK) solutions (3:1, 1h; 1:1; 1h; 1:3; 2h (v:v)) and in pure resin overnight. The embedding protocol was performed on ice. Final embedding was done in capsules which were subsequently incubated at $50^{\circ} \mathrm{C}$ for $3 \mathrm{~d}$.

\section{3. $\quad$ Preparation of the gold-enzyme complex and tissue labelling}

Cellulose and pectins were detected in plant tissues by enzymatic Au-labelling. Since our goal was to examine the location of such polysaccharides in isolated cuticles, but not determining their specific epitopes, the use of antibodies was not considered in this study. Hence, the commercial mixtures Celluclast and Pectinex, conjugated with $10 \mathrm{~nm}$ citratecoated Au nanoparticles ( 0.01\%; Sigma-Aldrich) were used. The Au-conjugated enzymes were prepared shortly before tissue labelling according to the methods described by Berg et al. [16] and Ferguson et al. [17], with some modifications. 
Briefly, $5 \mathrm{ml}$ of the colloidal Au solution were adjusted to $\mathrm{pH} 4.5$ by adding sodium hydroxide. Concerning cellulase, $1 \mathrm{ml}$ of the Au solution was mixed with $20 \mu \mathrm{l}$ of a $0.2 \%$ solution of the enzymatic mixture. A 2.5:1 colloidal Au:enzyme ratio was selected for preventing the flocculation of the Au colloid in the final solution [22]. After 5 min stirring of the Au-enzyme solution, $100 \mu \mathrm{l}$ of $1 \%$ polyethylene glycol (PEG, Sigma-Aldrich) were added in order to improve the stability of the Au-enzyme complex and to minimize non-specific enzyme adsorption to the tissues. The mixture was then centrifuged at 20,000 $\mathrm{g}$ for $1 \mathrm{~h}$ at room temperature. The resulting product had clear, supernatant and pink aqueous phases and a hard pink pellet. The clear aqueous phase was discarded and the pink one resuspended in 1 $\mathrm{ml}$ of $0.05 \mathrm{M}$ citrate buffer, $\mathrm{pH} 4.5$, with $0.1 \%$ PEG. Centrifugation and resuspension were repeated and the final Au-cellulase complex was stored at $4^{\circ} \mathrm{C}$ for further use. The same procedure was followed for preparing the Au-pectinase complex.

The $\mathrm{pH}$ value of the colloidal Au-enzymatic solution was selected taking into consideration the isoelectric points (pI) of the enzymes [23]. The most abundant enzyme produced by $T$. reesei is cellobiohydrolase I representing up to $60 \%$ of the total protein content secreted by the fungus [24]. The pI of this enzyme is 4.5-5.2 (lowest at 3.5; [25]). On the other hand, Pectinex contains several enzymes with pectolytic activities including polygalacturonase (product datasheet). As homogalacturonan may represent about $65 \%$ of pectins [26] and the main enzyme degrading its backbone is polygalacturonase, the $\mathrm{pI}$ of this enzyme (from $A$. aculeatus) was considered, being in the range 4.2-4.6 [27]. Therefore, all the solutions used for preparing Au-cellulase and -pectinase complexes were adjusted at pH 4.5.

Grids containing the resin embedded, ultra-thin sections to be labelled with Au-cellulase were carefully deposited onto drops of $10 \mu \mathrm{l}$ of citrate buffer plus $4 \%$ bovine serum albumin

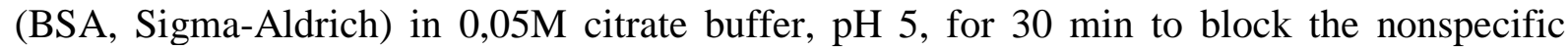
enzyme binding sites. The sections were then labelled with the Au-cellulase complex previously diluted at a 1:30 ratio in $0,05 \mathrm{M}$ citrate buffer, $\mathrm{pH} 5$, plus $1 \%$ BSA for $40 \mathrm{~min}$ at room temperature. The same procedure was followed for Au-pectinase tissue labelling, with the exception of the $6 \mathrm{pH}$ of citrate buffer solutions. The $\mathrm{pH}$ of the buffers was adjusted for enabling optimal activity of the enzyme mixtures when in contact with plant tissues (pH 5 for Celluclast [24]; pH 6 for Pectinex [28]). After labelling, sections were washed in citrate buffer (x1, $5 \mathrm{~min}$ ) and in bi-distilled water (x4, $5 \mathrm{~min}$ ).

\subsection{Control samples}

As control samples to determine the specificity of Au-enzyme complexes in binding to cellulose or pectins, leaves with intact cell walls and rat nerves were labelled following the procedures described above. Labelled and unlabelled leaf and cuticle samples were observed by TEM to assess whether the ultra-structure of the cuticle was influenced by the enzymatic hydrolysis and labelling protocol.

\subsection{Microscopy}

Gold-sputtered intact eucalypt, poplar and pear adaxial leaf surfaces, and the outer side of enzymatically digested adaxial cuticular membranes of leaves from the same species were examined with a Hitachi S-3400 N (Tokyo, Japan) and a Philips XL30 (Eindhoven, The Netherlands) scanning electron microscope (SEM).

For TEM observations, ultra-thin labelled and unlabelled tissue sections were mounted on nickel grids and examined with a Jeol 1010 electron microscope (Tokyo, Japan) equipped with a CCD Megaview camera, operated at $100 \mathrm{kV}$. Previously, labelled sections were poststained with 2\% aqueous uranyl acetate (EMS) and Reynolds lead citrate (EMS) for 3 and 1 
min, respectively. Unlabelled sections were post-stained with $2 \%$ aqueous uranyl acetate for 20 min and Reynolds lead citrate for 5 min.

\section{Results}

\section{1. $\quad$ Cuticle isolation and ultra-structure}

The adaxial leaf cuticles of eucalypt and pear were successfully isolated by means of cellulase and pectinase digestion. In contrast, just a few small-size leaf cuticle pieces of poplar could be obtained after enzymatic extraction, which were always attached to the outer layer of the cell wall or even to the entire cell wall of the epidermis.

The isolation process removed the wax nano-tubes of the EW layer of eucalypt leaves as confirmed by scanning electron microscopy observation of intact leaves and isolated cuticles (Fig. 1). However, the cuticle isolation process did not appear to alter the EW of poplar and pear leaves (data not shown). The cuticular ultra-structure of the remaining cuticular layer did not seem to vary after cuticle isolation (Figs. 2, 3).

A layer which may contain a high concentration of pectins was observed in the outer epidermal cell wall of eucalypt and especially of pear leaves being in direct contact with and extending into the cuticle (Fig. 2A, C). A granular layer in a similar position was observed in poplar leaves and cuticles (Figs. 2B, 3B, E), which contained cellulose and pectins as chemical constituents, as demonstrated by Au-labelling (data not shown).

\subsection{Location of cellulose and pectins in leaf cuticles}

Cellulose and pectins were found in all the digested cuticles, as confirmed by the presence of Au-conjugated cellulase and pectinase particles. Aggregates with variable degrees of density and single Au-enzyme particles were observed along the cuticle transversal sections (e.g., Fig. 3). However, a regular maximum distance from the bottom of the cuticle (in contact with the cell wall), was reached by most of the Au-enzyme particles in relation to the different plant species examined and micrographs representative for this phenomenon are shown in Fig. 3. For the three species, the labelling density was higher in the inner part of the cuticle for both cellulose and pectins, although most particles were not located on the fibrillar reticulum (data not shown). In all species, the highest rate of cellulose and pectin Au-labelling was always observed in the CL, while the outermost Au particles were identified in the cuticular region defined as the CP (data not shown). The density of Au particles present in each cuticle and/or cuticular region was not quantified since it was considered that the results would be biased by the presence of cuticular compounds which may mask the enzyme binding sites. In general, Au-cellulase particles were observed along the whole cuticle of poplar (Fig. 3B) and pear cuticles (Fig. 3C), while in eucalypt their presence was chiefly restricted to the internal CL (Fig. 3A). In contrast, pectins were mainly labelled almost along the whole cuticle and they were normally identified in positions closer to the EW layer for the three species (Fig. 3 D-F).

Evidence for the specificity of the labelling procedure used was gained from the presence of Au-enzyme particles on the cell walls of leaves, but not in other areas (excepting the cuticle), as well as their complete absence on rat tissues (Fig. 4). Gold-cellulase particles were found to be distributed along the cell wall, although they rarely appeared in the middle lamella and at epidermal cell corners, being these regions mainly labelled with Au-pectinase particles. 


\section{Discussion}

In this study, we analysed the location and gross kind of polysaccharides on leaf cuticle transversal sections of three species by means of enzymatic Au-labelling. The lack of preliminary information on the chemical composition of such leaf cuticles, led us to discard

the use of specific polysaccharide antibodies against the potential predominant cellulosic and pectic compounds or with different degrees of cross-linking.

The cuticles of eucalypt and pear leaves were obtained as intact layers after enzymatic digestion, in contrast to poplar cuticles, which broke into small pieces and remained attached at least to the outer layer of the epidermal cell wall.

A well-defined, dark layer probably associated with the middle lamella and hence containing pectins was observed at the epidermal cell wall-cuticle interface of pear, while a less conspicuous layer could be identified in eucalypt leaves (Fig. 2A, C). As stated before $[13,20]$, the existence of a pectin-rich layer along the cuticle and cell wall interface may ease cuticle separation by enzymatic hydrolysis.

Nevertheless, tissue digestion in cellulase and pectinase did not fully hydrolyse the granular layer of the poplar cell wall (Figs. 2B, 3B, E) possibly due to impregnation with nonhydrolysable and/or insoluble compounds. Gouret et al. [14] reported similar "black granulations" to those observed in the cell wall of poplar leaves (Fig. 2B) in the leaf cuticles of Galium aparine, and suggested that they might be mineral inclusions. However, Leppard and Colvin [29] associated the presence of cell wall granulates with different pectin forms. By means of Au-labelling, we gained evidence for the existence of cellulose and pectins in such cell wall granules of poplar cell walls. The presence of additional constituents (e.g., hemicelluloses), potential linkages among the compounds [30], or even cell wall lignification may yield these structures highly resistant to enzymatic digestion.

Cellulose and pectins were identified in the cuticle of the three species analysed, which indicates that the enzymes used for their isolation did not completely hydrolyse the polysaccharides present in the cuticles, possibly because they are protected from enzyme exposure [13] due to impregnation with non-hydrolysable and/or apolar cuticular constituents. The higher density of both Au-cellulase and -pectinase particles in the cuticle area next to the cell wall indicates a higher proportion of polysaccharides and/or higher enzyme accessibility and available surface area [31] in this zone. Similarly, Tenberge [18] observed cellulose labelling in the CL of spruce, but failed to detect pectins, maybe due to masking by other cuticular components.

The aggregation pattern of Au nanoparticles observed in all the samples (Fig. 3) corresponds to the general case of topological realizations of Au clusters described by Ghosh and Pal [32]. Most Au-cellulase particles were observed along the CL of poplar and pear cuticles, while in eucalypt it was restricted to the internal CL (Fig. 3A-C). In contrast, the majority of pectins was labelled along the whole CL for the three species (Fig. 3D-E). Cellulose and pectins were also identified in the CP and/or in the CP vicinity of the three species, though the density of gold particles considerably decreased compared to the CL, especially in the case of eucalypt. This finding contradicts the assumption of a polysaccharide-free CP [e.g., 7,8], and should be further investigated in future studies.

It must be stressed that despite the presence of Au-enzyme particles indicates the existence of cellulose or pectins in the labelled area, their absence does not imply a total lack of polysaccharides. The concentration of polysaccharides may gradually decrease or be jeopardized by other chemical compounds towards the outer regions of the cuticle as 
confirmed by Au-labelling. For example, the higher cutin content of the more external regions of the cuticle hypothesised by Wattendorf and Holloway [12] for Agave americana cuticles, may affect polysaccharide labelling by masking the binding sites of the enzymes.

The differences observed for eucalypt as compared to poplar and pear cuticles with regards to the position of Au particles, may be associated with the perennial versus deciduous leaf character. The eucalypt cuticle may have a higher proportion of waxes and cutin, and higher degree of lipid impregnation that may impede the access of the enzymes to polysaccharides.

By means of cellulose and pectinase Au-labelling, we were able to demonstrate the occurrence of cellulose and pectins in different areas of the leaf cuticle of the plant species analysed. The labelling of pectins along the cuticle in the three species makes us hypothesize their presence at early stages of cuticle ontogeny. It can be reckoned that pectins, which may constitute the first layer deposited in the plate of two daughter cells and are present in the middle lamellae of plant cells [33], may be preliminary excreted by epidermal cells shortly after division and prior to the formation of the pro-cuticle [8]. The cuticle would subsequently develop, having a structural frame provided by the existing pectin layer and the developing epidermal cell wall. Due to the deposition of new primary cell wall material towards the epidermal cell surface, the preliminary structural compounds may be allocated towards the external cuticle surface, with waxes being deposited on top owing to their lower surface free energy [34]. However, such primitive pectin band may be inhomogenously distributed due to strain forces suffered by cuticle and cell wall materials during cell enlargement.

The presence and distribution of polysaccharides in the leaf cuticles of the three species examined may lead to different rheological properties of such bio-materials in relation to environmental stimuli $[3,9]$ and may have a major structural role. Furthermore, the presence of cellulose and pectins maybe present as hydrogels [35] even in isolated cuticular membranes of plant organs, may actively contribute to the bi-directional transport of water and solutes, a possibility that has not been yet explored and will require future investigation.

\section{Contributions}

PG and VF designed the development of this study, carried out the experiments and wrote the manuscript. MLG and AF selected the tissue fixation and embedding TEM and Aulabelling protocols, and provided assistance with TEM observations. Discussion with LG and MK enabled to clarify the significance of the results in leaf anatomical, ontological and physico-chemical terms. Dynamic discussions between all authors facilitated the integration of the results following a multi-disciplinary approach. All authors contributed to improving the paper and approved the final manuscript.

\section{Acknowledgements}

We wish to thank Ramiro Martínez from Novozymes for providing free enzyme samples. PG is supported by a pre-doctoral grant from the Technical University of Madrid. VF is supported by a Ramón y Cajal contract (MINECO, Spain), co-financed by the European Social Fund. This study was supported by the Spanish Ministry of Economy and Competitiveness (MINECO, Spain; Project AGL2012-35580). 


\section{References}

[1] R. J. Carpenter, G. J. Jordan, A. Leigh, T. J. Brodribb, Giant cuticular pores in Eidothea zoexylocarya (Proteaceae) leaves, Am. J. Bot. 94 (2007) 1282-1288.

[2] J. J. Reina-Pinto, A. Yephremov, Surface lipids and plant defences, Plant Physiol. Biochem. 47 (2009) 540-549.

[3] E. Domínguez, J. A. Heredia-Guerrero, A. Heredia, The biophysical design of plant cuticles: an overview, New Phytol. 189 (2011) 938-949..

[4] F. Marga, A. Gallo, K. H. Hasenstein, Cell wall components affect mechanical properties: studies with thistle flowers, Plant Physiol. Biochem. 41(2003) 792-797.

[5] R. A. Burton, M. J. Gidley, G. B. Fincher, Heterogeneity in the chemistry, structure and function of plant cell walls, Nature Chem. Biol 6 (2010) 724-732.

[6] M. Bernal, L. Llorens, R. Julkunen-Tiitto, J. Badosa,, D. Verdaguer, Altitudinal and seasonal changes of phenolic compounds in Buxus sempervirens leaves and cuticles, Plant Physiol. Biochem. 70 (2013) 471-482.

[7] H. von Mohl, Untersuchungen der Frage: bildet die Cellulose die Grundlage sammtlicher Vegetabilischen Membranen. Botan Zeitung 5 (1847) 497-505.

[8] C. E. Jeffree, The fine structure of the plant cuticle. In: Riederer M, Müller C, eds. Annual Plant Reviews, vol. 23, Biology of the plant cuticle. Oxford: Blackwell Publishing (2006), pp. 11-125.

[9] G. López-Casado, A. J. Matas, E. Domínguez, J. Cuartero, A. Heredia, Biomechanics of isolated tomato (Solanum lycopersicum L.) fruit cuticles: the role of the cutin matrix and polysaccharides, J. Exp. Bot. 58 (2007) 3875-3883.

[10] E. Domínguez, A. Heredia, Water hydration in cutinized cell walls: a physico-chemical analysis, Biochimica et Biophysica Acta 1426 (1999) 168-176.

[11] E. J. Johnson, O. Dorot, J. Liu, B. Chefetz, B. Xing, Spectroscopic characterization of aliphatic moieties in four plant cuticles. Commun. Soil. Sci. Plant Anal. 38 (2007) 24612478.

[12] J. Wattendorff, P. J. Holloway, Studies on the ultrastructure and histochemistry of plant cuticles: isolated cuticular membrane preparations of Agave americana L. and the effects of various extraction procedures, Ann. Bot. 49 (1982) 769-804.

[13] R. F. Norris, M. J. Bukovac, Structure of the pear leaf cuticle with special reference to cuticular penetration, Am. J. Bot. 55 (1968) 975-983.

[14] E. Gouret, R. Rohr, A. Chamel, Ultrastructure and chemical composition of some isolated plant cuticles in relation to their permeability to the herbicide diuron, New Phytol. 124 (1993) 423-431.

[15] A. J. Matas, E. D. Cobb, J. A. Bartsch, D. J. Paolillo, K. J. Niklas. 2004. Biomechanics and anatomy of Lycopersicon esculentum fruit peels and enzyme-treated samples, Am. J. Bot. 91 (2004) 352-360.

[16] R. H. Berg, G. W. Erdos, M. Gritzali, R. D. Brown, Enzyme-gold affinity labelling of cellulose, J. Electron Microscop. Tech. 8 (1988) 371-379.

[17] C. Ferguson, T. T. Teeri, M. Siika-aho, S. M. Read, A. Bacic, Location of cellulose and callose in pollen tubes and grains of Nicotiana tabacum, Planta 206 (1998) 452-460.

[18] K. B. Tenberge, Ultrastructure and development of the outer epidermal wall of spruce (Picea abies) needles, Canad. J. Bot. 70 (1992) 1467-1487.

[19] L. Schreiber, J. Schönherr, Water and solute permeability of plant cuticles: measurement and data analysis. Heidelberg: Springer (2009). 
[20] W. H. Orgell, The isolation of plant cuticle with pectic enzymes, Plant Physiol. 30 (1955) 78-80.

[21] A. W. Robards, A. J. Wilson, eds. Procedures in electron microscopy. Chichester: Wiley (1993).

[22] C. de Roe, P. J. Courtoy, P. Baudhuin, A model of protein-colloidal gold interactions, J. Histochem. Cytochem. 35 (1987) 1191-1198.

[23] H. Essa H, E. Magner, J. Cooney, B. K. Hodnett, Influence of pH and ionic strength on the adsorption, leaching and activity of myoglobin immobilized onto ordered mesoporous silicates., J. Molec. Catalysis B: Enzymatic 49 (2007) 61-68.

[24] L. Rosgaard, Enzymatic hydrolysis of pretreated barley and wheat straw. PhD Thesis, Technical University of Denmark, Denmark (2007).

[25] Z. Xu, Y. Miao, J. Y. Chen, X. Jiang, L. Lin, P. Ouyang, Co-immobilization mechanism of cellulase and xylanase on a reversibly soluble polymer, Appl. Biochem. Biotech. 163 (2011) 153-161.

[26] D. Mohnen, Pectin structure and biosynthesis, Curr. Op. in Plant Biol. 11 (2008) 266277.

[27] S. W. Cho, S. Lee, W. Shin, The X-ray structure of Aspergillus aculeatus polygalacturonase and a modeled structure of the polygalacturonase-octagalacturonate complex, J. Molec. Biol. 311 (2001) 863-878.

[28] A. Tanriseven, Y. Aslan Y, Immobilization of Pectinex Ultra SP-L to produce fructooligosaccharides. Enzyme Microb Techn 36 (2005) 550-554.

[29] G. G. Leppard, J. R.Colvin, Electron-opaque fibrils and granules in and between the cell walls of higher plants, J. Cell Biol. 53 (1972) 695-703.

[30] D. J. Cosgrove, Growth of the plant cell wall, Nature Rev. Molec. Cell Biol. 6 (2005) 850-861.

[31] S. D. Mansfield, C. Mooney, J. N. Saddler, Substrate and enzyme characteristics that limit cellulose hydrolysis. Biotechnol Progr 15 (1999) 804-816.

[32] S. K. Ghosh, T. Pal, Interparticle coupling effect on the surface plasmon resonance of gold nanoparticles: from theory to applications, Chem. Rev. 107 (2007) 4797-4862.

[33] J. P. Vincken, H. A. Schols, R. J. F. J. Oomen, M. C. McCann, P, Ulvskov, A. G. J. Voragen, R. G. F. Visser, If homogalacturonan were a side chain of rhamnogalacturonan I. Implications for cell wall architecture, Plant Physiol. 132 (2003) 1781-1789.

[34] M. Khayet, V. Fernández, Estimation of the solubility parameters of model plant surfaces and agrochemicals: a valuable tool for understanding plant surface interactions, Theor. Biol. Med. Mod. 9 (2012) 45.

[35] M. A. Zwieniecki, P. J. Melcher, N. M. Holbrook, Hydrogel control of xylem hydraulic resistance in plants, Science 291 (2001) 1059-1062. 


\section{Figures}

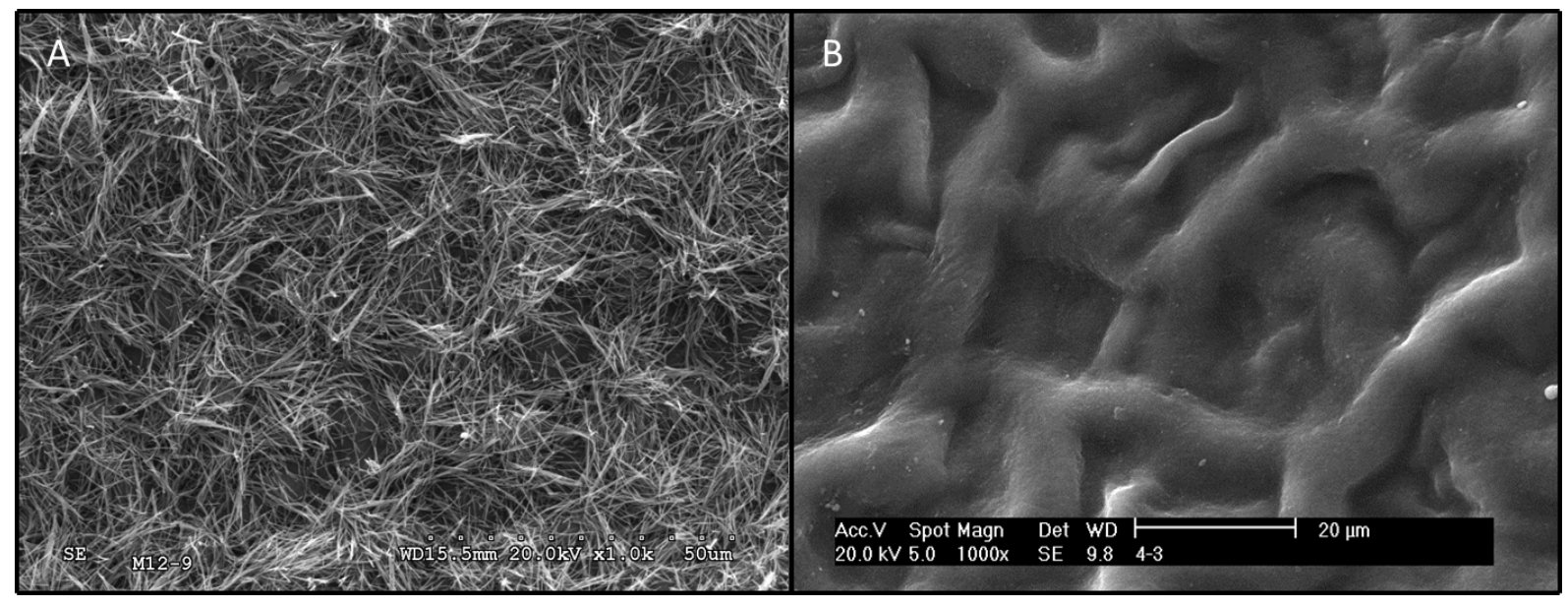

Fig. 1. Influence of enzymatic digestion on the outer surface of eucalypt adaxial cuticle (B) as compared to intact the intact leaf (A), which removed the epicuticular wax nano-tubes. Magnification: x1000. 


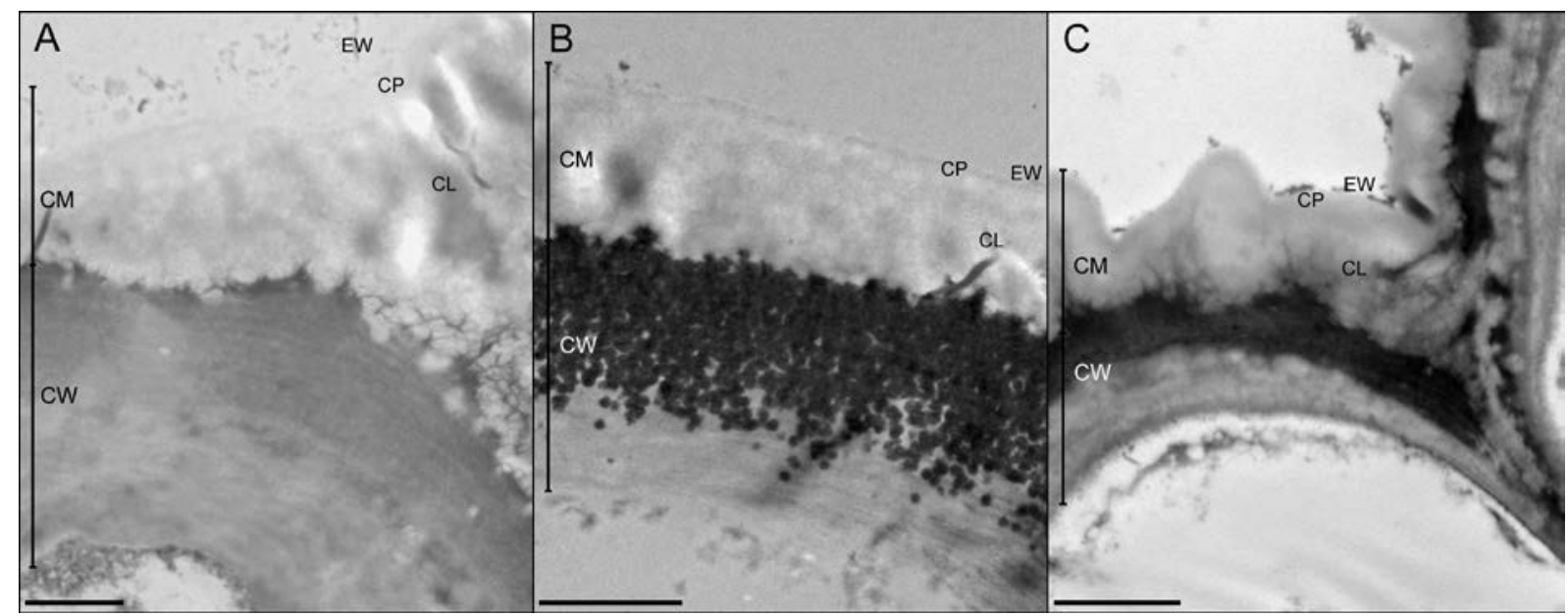

Fig. 2. Transversal sections of the adaxial cuticle and outer cell wall of intact leaves of eucalypt (A), poplar (B) and pear (C). The different layers according to Jeffree [8] are referred to as: CL: cuticular layer; CM: cuticular membrane; CP: cuticle proper; $\mathrm{CW}$ : cell wall; $\mathrm{EW}$ : epicuticular wax layer. Bars $=2 \mu \mathrm{m}$. 


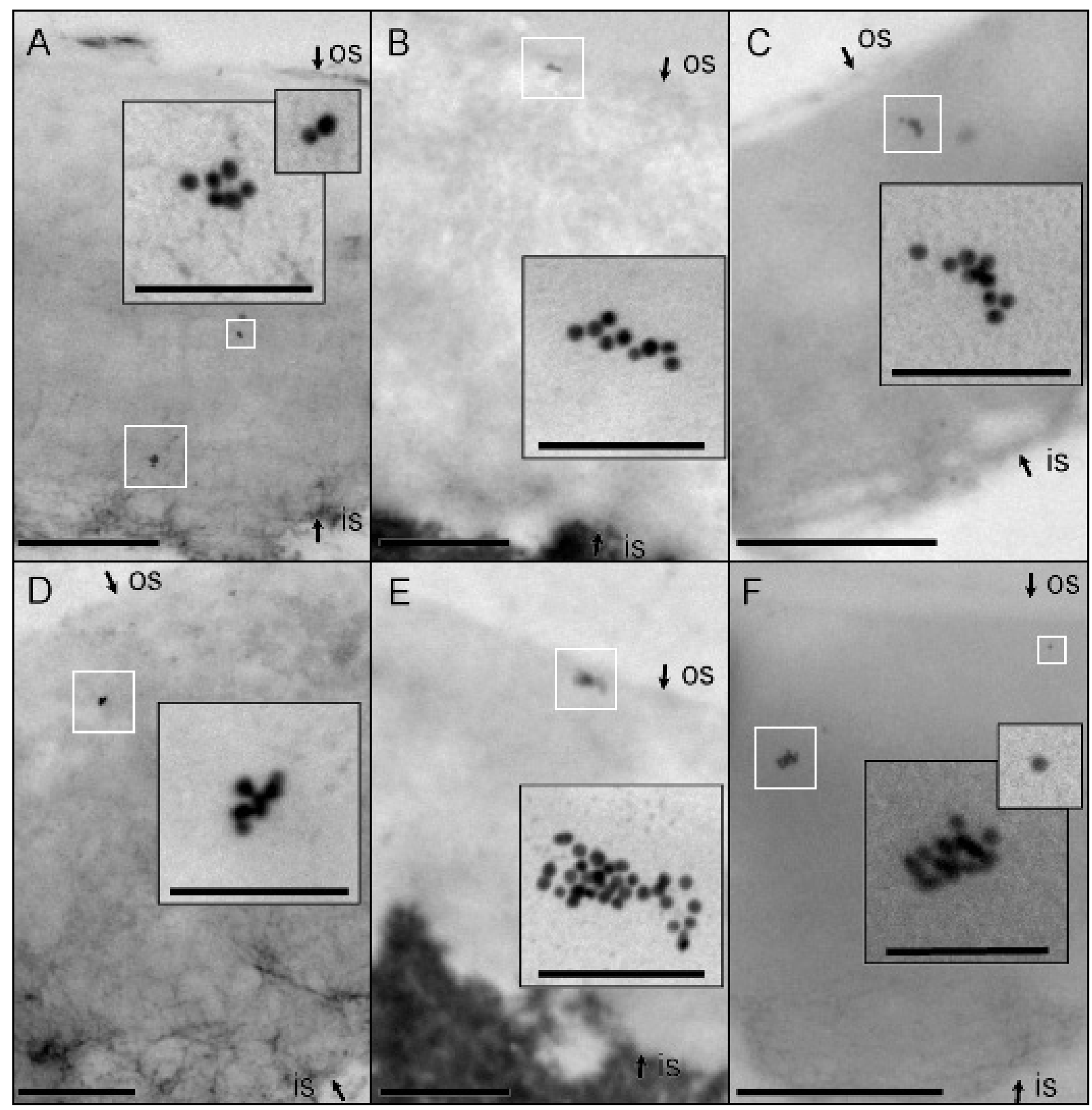

Fig. 3. Location of cellulose and pectins in enzymatically digested cuticles of eucalypt, poplar and pear by means of Au-cellulase (A, B, C) and Au-pectinase (D, E, F) particles. Arrows indicate the limits of the cuticle. IS: inner side (of the cuticle); OS: outer side (of the cuticle). Bars $=200 \mathrm{~nm}$. Inset bars $=100 \mathrm{~nm}$. 


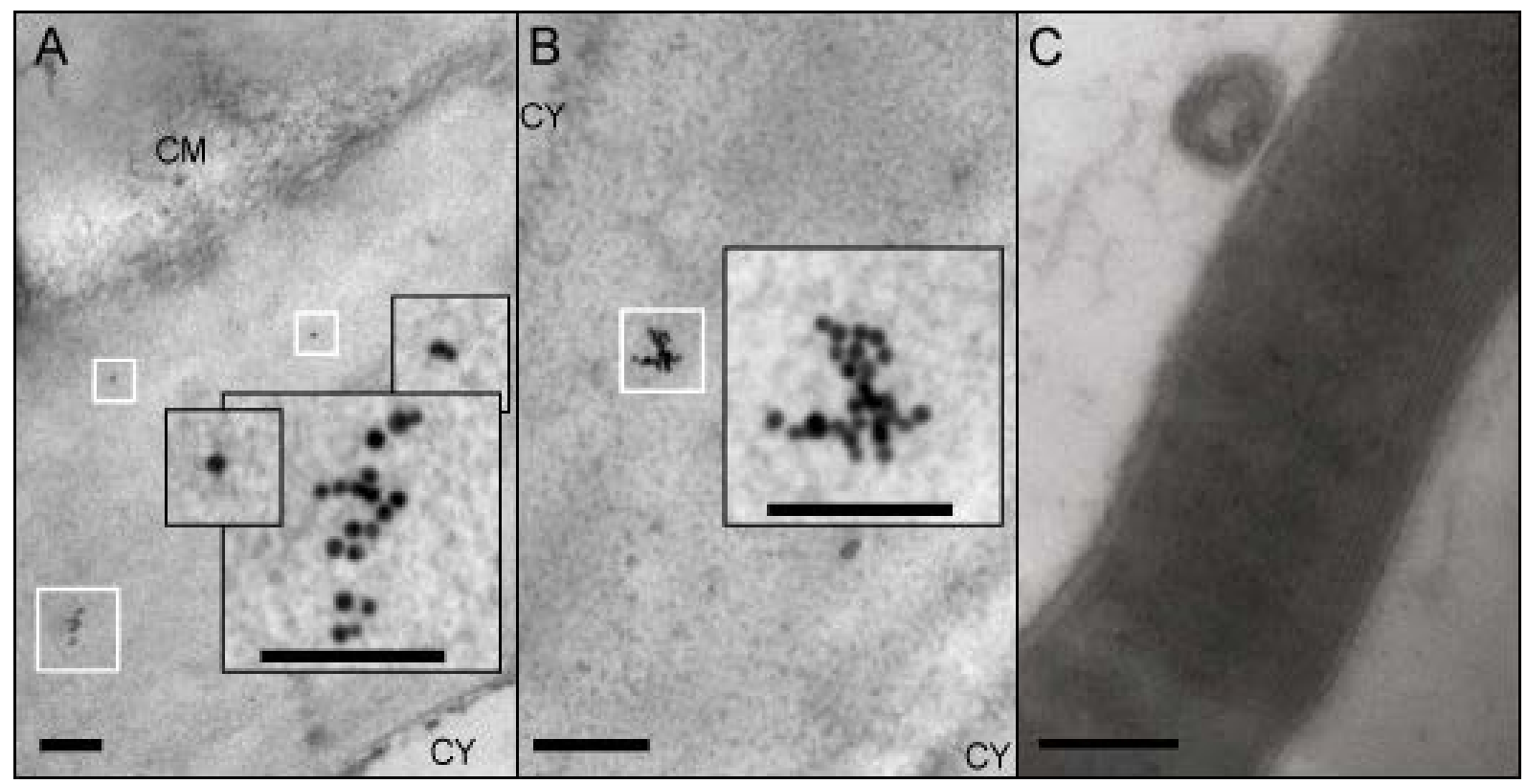

Fig. 4. Control samples for Au-labelling. Eucalypt cell wall labelled with Au-cellulase (A) and Au-pectinase (B; middle lamella region) and rat nerves labelled with Au-cellulase (C; absence of gold particles, as in the samples labelled with Au-pectinase). CM: cuticular membrane, CY: cytoplasm. Bars= $200 \mathrm{~nm}$. Inset bars $=100 \mathrm{~nm}$. 